\title{
COVID-19 vaccines: where we stand and challenges ahead
}

\author{
Guido Forni ${ }^{1}$ Alberto Mantovani $\mathbb{1}^{2,3} \cdot$ on behalf of the COVID-19 Commission of Accademia Nazionale dei Lincei, \\ Rome
}

Received: 12 November 2020 / Revised: 16 December 2020 / Accepted: 16 December 2020 / Published online: 21 January 2021

(c) The Author(s), under exclusive licence to ADMC Associazione Differenziamento e Morte Cellulare 2021

\begin{abstract}
In the eleven months elapsed since the identification of the SARS-CoV-2 virus and its genome, an exceptional effort by the scientific community has led to the development of over 300 vaccine projects. Over 40 are now undergoing clinical evaluation, ten of these are in Phase III clinical trials, three of them have ended Phase III with positive results. A few of these new vaccines are being approved for emergency use. Existing data suggest that new vaccine candidates may be instrumental in protecting individuals and reducing the spread of pandemic. The conceptual and technological platforms exploited are diverse, and it is likely that different vaccines will show to be better suited to distinct groups of the human population. Moreover, it remains to be elucidated whether and to what extent the capacity of vaccines under evaluation and of unrelated vaccines such as BCG can increase immunological fitness by training innate immunity to SARS-CoV-2 and pathogenagnostic protection. Due to the short development time and the novelty of the technologies adopted, these vaccines will be deployed with several unresolved issues that only the passage of time will permit to clarify. Technical problems connected with the production of billions of doses and ethical ones connected with the availably of these vaccines also in the poorest countries, are imminent challenges facing us. It is our tenet that in the long run more than one vaccine will be needed to ensure equitable global access, protection of diverse subjects and immunity against viral variants.
\end{abstract}

\section{Facts}

- The COVID-19 outbreak has prompted scientists from around the world to design anti-SARS-CoV-2 vaccines.

- The free availability of basic science data has allowed creating vaccines based on very innovative platforms and directed towards very well defined sensible targets.

Edited by T. Mak

Members of the COVID-19 Commission of Accademia Nazionale dei Lincei, Rome are listed below Acknowledgements

Alberto Mantovani

alberto.mantovani@humanitasresearch.it

1 Accademia Nazionale dei Lincei, Via della Lungara 10, 00165 Rome, Italy

2 Istituto Clinico Humanitas IRCCS, Humanitas University, via Rita Levi Montalcini 4, 20090 Pieve Emanuele, Milan, Italy

3 William Harvey Research Institute, Queen Mary University of London, Charterhouse Square, London EC1M 6BQ, UK
- High financial support both from private consortia and the governments of several nations have made it possible to develop the new vaccines extremely quickly.

- The possibility of having numerous vaccines based on different technologies will allow us to select those that can be most effective in specific phases of the pandemic and different parts of the world.

- The production and distribution of billions of doses of COVID-19 vaccines is the new, difficult challenge.

- The creative and technological effort that lead to the development of COVID-19 vaccines has changed the way of thinking and designing new vaccines for other diseases.

\section{Open questions}

- Will the new vaccines be able to control the COVID-19 pandemic?

- Will there be vaccines that will be able to protect the most fragile sections of the human population?

- The development of vaccines in a very short time necessarily implies that is not yet possible to know their long-term efficacy and possible side effects. 
- Will it be possible to overcome financial and political problems and allow COVID-19 vaccines to be available with equity for the entire population of the world?

\section{Introduction}

The hope and hype that the media and public at large are placing on having as soon as possible a vaccine that protects against COVID-19 is the result of the great triumphs that vaccines have had and are having in the control of infectious diseases. However, there is a long series of infectious diseases in which vaccines are only partially effective and we have a series of sensational vaccine defeats [1]. Indeed, each disease is an immunological problem in itself: even today, with all the data at one's disposal, it is difficult to predict what kind of vaccine can be truly effective. This difficulty is even greater for COVID-19, a new disease in which ongoing studies in laboratories worldwide are adding new data at a tremendous pace. SARS-CoV2, the coronavirus responsible for COVID-19 is an RNA virus, and these viruses generally have a high mutation rate. Genetic instability has long been considered to represent a challenge to develop effective vaccines against RNA viruses.

In many cases, recovery from a viral disease rests on the combined action of antibodies in the biological fluids that neutralize the viral particles and the killer activity of lymphocytes that track down and kill virus-infected cells. However, there are viral diseases whose healing depends mainly, if not exclusively, on the antibody response and others where the destructive action of the killer lymphocytes is fundamental. What is the case with COVID-19 is not yet clearly defined although several data suggest that the major protective effect is to be attributed to antibodies against the Spike protein and in particular against its receptor-binding domain. Often, healed patients display high titers of SARS-CoV-2 neutralizing antibodies [2]. Data on the role of mucosal immunity and secretory IgA and IgM are scarce. Furthermore, we cannot yet know how long the protection acquired by recovered patients will last. This point is of interest since often, the duration of the protection after healing somewhat corresponds to the duration of the protection provided by the vaccine.

Despite the impressive amount of studies carried out since the virus was first characterized, there are still a large number of unknowns about this disease. And it is precisely these unknowns that fully justify the very different conceptual and technological strategies that are currently pursued in the preparation of vaccines against COVID-19. This diversification appeared essential precisely because, for many diseases, but particularly for a new disease as COVD19 , it is difficult to predict which type of immune response and therefore vaccine will be more effective.
As shown by Lurie et al. [3], there are major differences between traditional vaccine development and development under the pressure of a rampant epidemic. Because of the pressure created by the pandemic, multiple activities are carried at financial risks, without knowing whether the candidate vaccine will be safe and effective, including very early manufacturing and scale-up to commercial scale before the establishment of clinical proof of concept. However, in many cases, the financial risk is mainly being taken by Public Institutions and Governments which provide financial support to companies in their development effort.

\section{Technological platforms: the bright side of human creativity}

As of December 2020, just eleven months after the definition of the SARS-CoV-2 genome, there are over 150 official vaccine projects $[4,5]$. About fifty of them have already reached human experimentation and a few of these are currently administered to some sectors of the general population. By exploiting different technologies, these anti-SARS-CoV-2 candidate vaccines are targeting the whole SARS-CoV-2, molecules or fragments of molecules expressed on this virus surface. These different candidate vaccines can be grouped based on the technological platform exploited to elicit a protective immune response. However, almost every vaccine project has its peculiarities that make it unique and which could have significant consequences regarding the efficacy or duration of the induced protection or the safety of the vaccine. In Figs. 1, 3, 4 and 5 details of selected vaccine projects that are currently in Phase III trial are shown.

Faced with this variety of projects and the determination and speed with which they are carried out, one cannot help but be amazed by human scientific creativity. Even if these various projects compete with each other and have their technical secrets, their formulation comes from basic scientific research that is structured as open cooperation between all laboratories in the world. Indeed, the numerous freely available basic research data on the mechanisms of SARS-CoV-2 infection have convinced most developers of innovative vaccine to focus their efforts on inducing an immune response against the Spike protein (Fig. 2). The new data emerging from Phase III studies show that vaccines based on nucleic acids coding for the Spike protein, carried by liposomes or adenoviruses, can elicit an effective protective response [6-9]. These four impressive press releases provide the first evidence that vaccines based on previously unproven technologies can significantly contribute to the control of a pandemic that to date has caused more than 1.5 million deaths [10]. 
Fig. 1 Twelve candidate vaccines currently in Phase III trial. COVID-19 vaccines based on the whole inactivated SARSCoV-2.

Virus SARS-COV-2

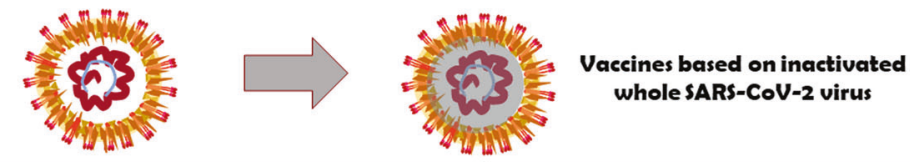

\begin{tabular}{|c|c|c|c|}
\hline Company and country & $\begin{array}{l}\text { Vaccine } \\
\text { name }\end{array}$ & $\begin{array}{l}\text { Number of } \\
\text { doses }\end{array}$ & Approval and registration \\
\hline Sinovac Biotech, China & -- & 2 & $\begin{array}{l}\text { Ahead of Phase II trial it is offered to essential workers and } \\
\text { other high-risk people of the Chinese town of Jiaxing for } \\
\text { about } 30 € / \text { dose }\end{array}$ \\
\hline $\begin{array}{l}\text { Beijing Institute of Biological } \\
\text { Products and Sinopharm, } \\
\text { China }\end{array}$ & BBIBP-CorV & 2 & $\begin{array}{l}\text { Limited approval for Chinese health care workers and } \\
\text { ahead of Phase III trial. This vaccine has been approved } \\
\text { by United Arab Emirates on the basis of preliminary data } \\
\text { showing that it is } 86 \% \text { effective. }\end{array}$ \\
\hline $\begin{array}{l}\text { Wuhan Institute of } \\
\text { Biological Products, } \\
\text { and Sinopharm, China }\end{array}$ & --- & 2 & $\begin{array}{l}\text { Limited approval for Chinese health care workers and } \\
\text { soldiers ahead of Phase III trial. }\end{array}$ \\
\hline Bharat Biotech, India & COVAXIN & 2 & $\begin{array}{l}\text { The Phase III trial with } 26000 \text { volunteers is expected to } \\
\text { close in February } 2021 .\end{array}$ \\
\hline
\end{tabular}

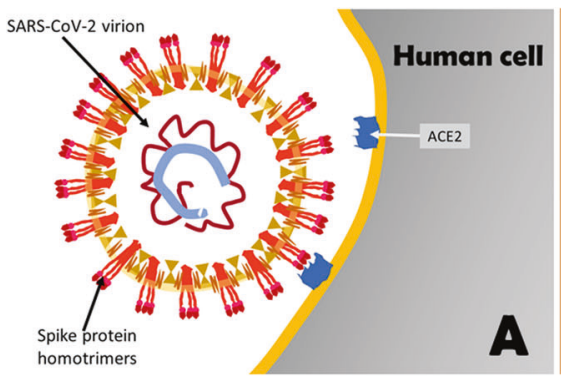

Fig. 2 Spike as a target for vaccine development. Within a few months after the identification of the new SARS-CoV-2, the freely available data made by numerous laboratories around the world provided a defined picture of the virus structure and of the steps of human cell infection. A SARS-CoV-2 is an oily spherical particle containing a single-stranded positive-sense RNA of about $30 \mathrm{~kb}$ wrapped and coiled by the Nucleocapsid protein. The virus outer shell consists of three other structural glycoproteins: Spike, Envelope, and Membrane, and a lipid coating. On the surface of SARS-CoV-2, three Spike glycoproteins aggregate protrudes outside the pericapsid and may interact at a high affinity with Angiotensin-Converting Enzyme 2

It will be important to compare the intensity and duration of the protective responses elicited by these four vaccines with those induced by vaccines based on technological platforms that are slower to develop. Other vaccine candidates that are coming might work better than the first, provide a longer immunity, while other factors such as cost or side effects might offer benefits to specific groups, such as the elderly, pregnant women, or people in low-income countries [11, 12].

\section{Vaccines based on attenuated SARS-CoV-2 viruses}

The history of vaccination begins with vaccines based on a living microbe that has been weakened so it can not cause

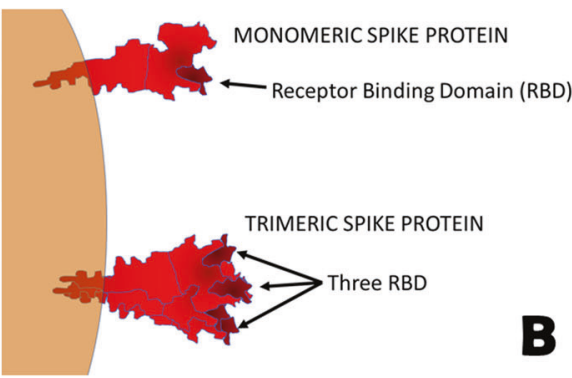

(ACE2), an exopeptidase normally present on the outer surface of a wide variety of human cells. B The Spike protein consists of two domains, S1 and S2. In the most external domain, a region known as Receptor-Binding Domain (RBD), allows the high-affinity binding of the SARS-CoV-2 to the N-terminal domain of the ACE2. The progressive elucidation of the critical role of this interaction provided the key insights that spurred several developers of innovative vaccine to target the Spike protein and its RBD $[2,66]$. The recent reports on the protective efficacy of vaccines based on different platforms targeting the Spike protein [6-9] suggest that the freely available basic science data allowed to make a winning bet [10].

disease. Since attenuated microbes retain the ability to replicate in vivo giving rise to a limited disease, they are very effective in stimulating the immune system and inducing a strong and persistent immune memory that is efficacious in preventing infection. Hundreds of millions of people have been protected from disabling and fatal diseases by using attenuated vaccines [1].

\section{Strategy}

This is the most traditional technology exploited in the construction of vaccines. Live attenuated vaccines can be obtained by growing the virus in unfavorable conditions or by generating a genetically weakened version of the virus. However, the attenuation of trillions of viruses is complex 
and delicate and can be associated with major biosafety risks [13]. Once produced, their storage and handling require carefully observed procedures.

The experience with attenuated virus vaccines shows that rare but significant side effects could be expected since attenuated viruses cause disease, even if this is a minor one. The oral route (as in the case of the Sabin polio vaccine) and the intranasal route could induce a mucosal immunity based on secretory $\operatorname{IgA}$ and $\operatorname{IgM}$.

\section{Frontrunners}

Only three projects of attenuated SARS-CoV-2 vaccines are in active preclinical development at the following institutions:

- The Serum Inst of India, India, in collaboration with Codagenix, a New York private biotech;

- Indian Immunologicals Ltd, India, in collaboration with the Griffith University, Australia;

- Mehmet Ali Aydunar Univ, Turkey.

None of these vaccine projects have yet reached the stage of clinical trials.

\section{Vaccines based on the inactivated SARS-CoV-2 viruses}

Vaccines based on killed microorganisms (inactivated vaccines) belong to a very traditional technological platform that has led to numerous vaccines. The vaccines produced using this method are more stable than live attenuated vaccines but their limit is mainly related to the short duration of immune memory which demands inoculation of higher amounts of vaccine or the association of the inactivated microorganism with an adjuvant. The immune response elicited is directed not only against the Spike protein but also against many other SARS-CoV-2 antigens. While the induced response is generally weaker concerning that induced by attenuated viruses, the vaccine is more easily handled, less expensive, and much safer.

\section{Strategy}

The SARS-CoV-2 is inactivated by exploiting different chemical techniques. All these candidate vaccines are injected intramuscularly.

\section{Frontrunners}

Seven vaccine candidates based on variously inactivated SARS-CoV-2 virions are in clinical trials, four of which in
Phase III trials and already approved for limited use (Fig. 1). When available, reports from Phase II trials suggest that the vaccine is safe and induces a high titer of antibodies. The seven clinical trials are run by:

- Sinovac Biotech, China, this vaccine called CoronaVac is in late-stage Phase III trial and interim results are expected in late November. Meanwhile, CoronaVac has already been approved for limited use among the general population (Fig. 1);

- Sinopharm, China, two of its distinct projects are approved for limited use in the general population (Fig. 1);

- Wuhan Inst Biol Products, China, this vaccine has been approved for limited use in the general population (Fig. 1);

- Chinese Acad Med Sci, China;

- Bharat Biotech, India, this vaccine, called Covaxin, is in late stage Phase III trial;

- RIBSP, Kazakhstan.

\section{Vaccines based on SARS-CoV-2 proteins}

There are several human vaccines based on proteins present on the surface of microbes [1]. Initially, these proteins were purified from the microbes while today, in most of the cases, they are produced in vitro exploiting the recombinant DNA technology.

\section{Strategy}

The large trimeric aggregates of the Spike protein that protrude outside the virion play an essential role in the docking of the SARS-CoV-2 to human cells. Therefore, the Spike protein or its fragments are the targets of all these vaccines even if in a few cases other SARS-CoV-2 proteins -mostly the nucleoprotein (N)- are also targeted. To activate a robust immune response, often these vaccines exploit adjuvants, either of bacterial or synthetic origin.

\section{Frontrunners}

There are very numerous vaccine projects based on SARSCoV-2 proteins, their fragments, or their fragments combination. At least sixteen candidate vaccines are already in human trials and two in Phase II trial (Fig. 3):

a. Spike protein or its fragments plus adjuvant Adimmune, Taiwan; Bektop, Russia; Biotechnology Vector, Russia; Clover Biopharmarm plus GSK adjuvant, China-Italy;

CoVaxx, US; Inst Finlay de Vacuna Vaccine, Cuba plus adjuvant; Medigen, Taiwan-US, plus CpG adjuvant; 
Fig. 3 Twelve candidate vaccines currently in Phase III trial: COVID-19 Vaccines based on Spike protein. These vaccines are probably the most immunogenic also thanks to the combination with new adjuvants [14]. The exploitation of transgenic plants as producers of Spike protein could allow the production of large quantities of the vaccine at an extremely low cost.

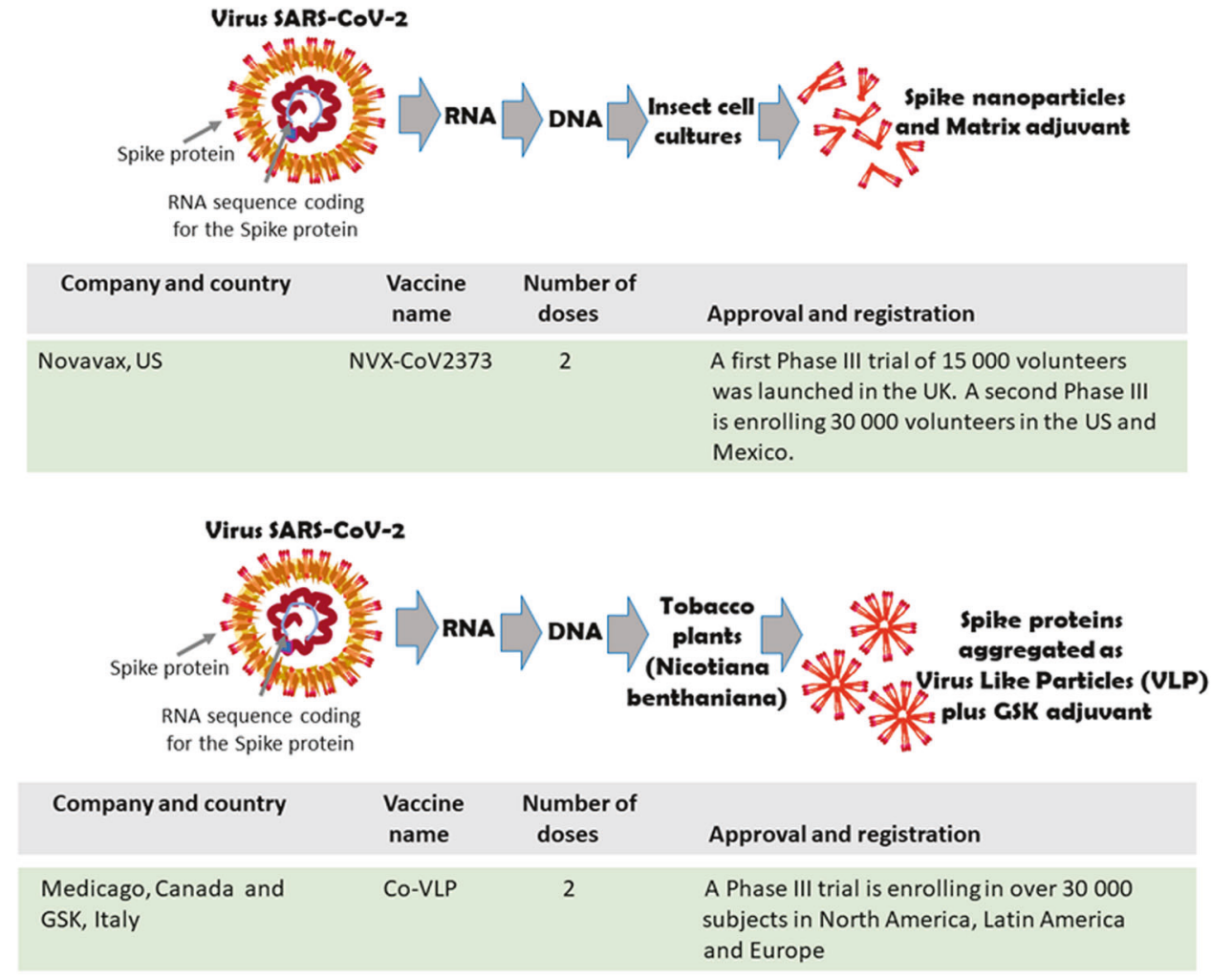

Sanofi plus GSK adjuvant, France - Italy;

The Univ of Queensland, Australia;

Univ Tübingen, Germany;

Vaxine, Australia, plus adjuvant;

West China Hosp Sichuan Univ., China;

ZFSW Anhui Zhifei Longcom, China, plus adjuvant.

b. Proteins carried by nanoparticles

Novavax, US, US, Australia, and South Africa, plus adjuvant (Fig. 3).

c. Oral tablet containing Spike protein fragments Vaxart, US.

d. Microneedle skin patch delivering Spike proteins Univ Queensland, Australia

e. Spike protein or its fragments inserted in virus-like particles (VLP)

SpyBiotech/Serum Institute of India, India.

f. Tobacco plant-produced proteins

Kentucky Bio Processing, US.

g. Tobacco plant-produced proteins in virus like particles (VLP)
Medicago plus GSK adjuvant, US - Italy (Fig. 3).

\section{Naked DNA-based vaccines}

The DNA and mRNA-based platforms offer great flexibility in terms of manipulation of the coded antigen and great potential for speed. Currently, there are no DNA vaccines registered for human use; however, DNA vaccines are commonly used in veterinary medicine. These vaccines are stable and can easily be produced in large amounts in bacteria.

\section{Strategy}

Once injected into the muscle or skin, DNA plasmids enter human cells, and their ability to enter may be enhanced by a very short local electrical pulse (electroporation). Once entered, plasmid DNA induces the cell to produce temporarily the target protein. In this way, DNA vaccination stimulates the production of antibodies and the activation of killer $\mathrm{T}$ cells.

\section{Frontrunners}

Six DNA vaccines are entering human trials. All code the Spike protein or its fragments.

a. Naked DNA plasmids 
Zydus Cadila, India;

AnGes, Japan;

Takis, Italy.

b. Naked DNA plasmids plus electroporation Inovio, US;

Genexine, Korea;

Karolinska Inst, Sweden + Inovio, Italy.

\section{mRNA-based vaccines}

While messenger RNA (mRNA) has not yet produced any registered vaccine, several vaccine projects exploit this technology for the creation of SARS-CoV-2 vaccines. Unlike DNA, RNA must be transported in various ways to enter the human cell. Once entered, the mRNA vaccine temporarily induces the cell to produce the antigen protein coded by the mRNA.

\section{Strategy}

In most of these vaccine projects, the mRNA is carried by lipid microvesicles (liposomes). Also, in the case of anti-SARS-CoV-2 mRNA vaccines, the target antigen coded by the mRNA is mostly if not only, represented by the Spike protein, its variants, or its fragments. These vaccine preparations have to be kept at -30 to $-80^{\circ} \mathrm{C}$.

\section{Frontrunners}

There are many vaccine projects based on mRNA and its variants coding the Spike protein, its variants, or its fragments. Two of those have finished Phase III trials (Fig. 4). The vaccine mRNA may be carried by:

a. Lipid vesicles (Liposomes)

Abogn, China;

CureVac, Germany;

Moderna, US (Fig. 4);

Pfizer, US - BioNTech, Two candidate vaccines were tested in parallel, and one finished Phase III trial (Fig. 5);

Univ Oxford, UK (Fig. 4) An inhaled form of the vaccine is also tested but has not yet reached Phase III trial.

b. Nanoparticles

Arcturus Ther, Singapore.

\section{Vaccines based on viral vectors}

The DNA coding for the Spike protein can be conveyed into the cells by viral vectors. By inserting the DNA in a virus, it is possible to exploit the virus's great ability to infect and deliver the mRNA into the human cells.

\section{Strategy}

The virus inside which the DNA is inserted may lose its ability to replicate. Since a preexisting immunity against the virus vector may affect vaccine efficacy, primate viruses (from chimpanzee, gorilla...) are often exploited as vectors. In other cases, the DNA is inserted into replication active virus vectors: as these viruses can propagate to
Fig. 4 Twelve candidate vaccines currently in Phase III trial: COVID-19 Vaccines based on Spike protein mRNA carried by lipidic

microparticles. These vaccines were the first to be developed and tested on volunteers. At the time of writing the Pfizer BioNTech BNT162b2 has been cleared by UK regulatory authorities. This "historical" vaccine approval paves the way for mass immunization in Western countries.

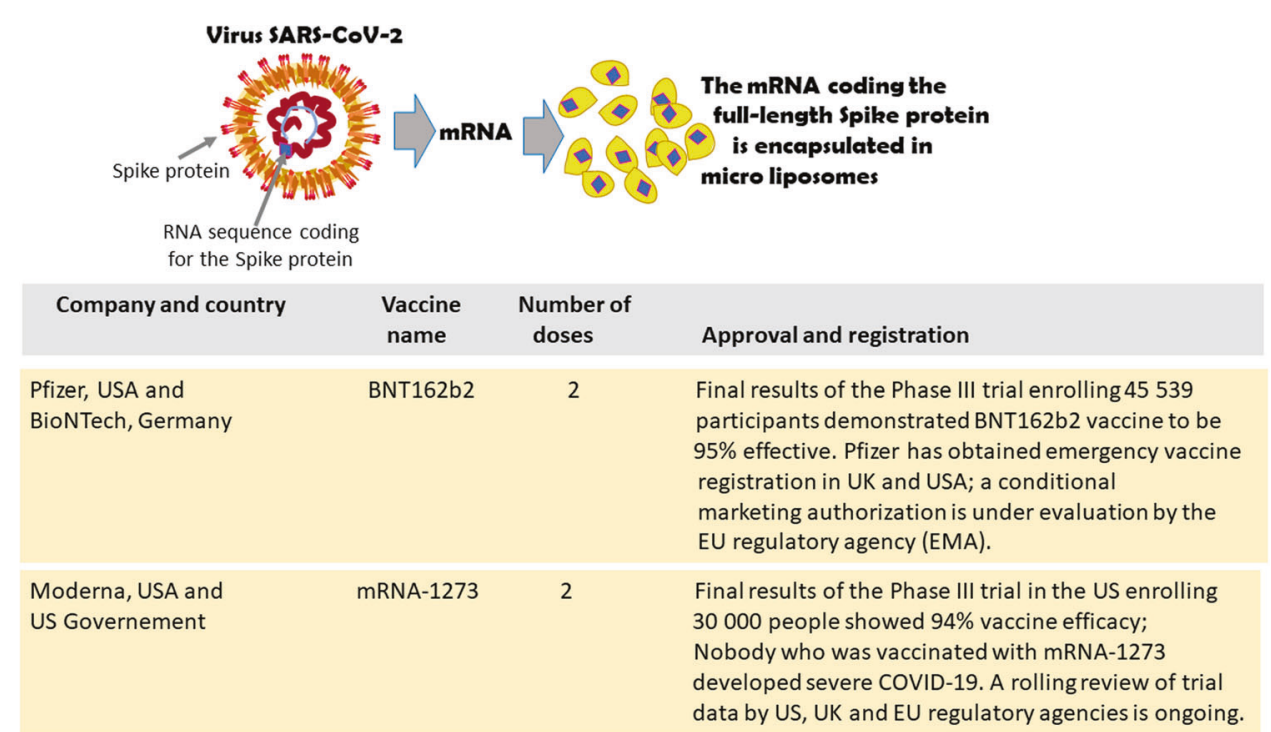


Fig. 5 Twelve candidate vaccines currently in Phase III trial. COVID-19 Vaccines based on Spike protein DNA carried by adenoviruses. The Chinese Can Sino Ad5-nCoV and the Russian Gamaley Res. Inst. Sputnik V vaccines have already obtained a limited authorization and have been administered to sections of the population. Despite the excellent preliminary results and welldocumented immunogenicity, the Phase III trial on AstraZeneca/University of Oxford ChAdOx1 vaccine provided provocative but somewhat contradictory results that require further study [8]

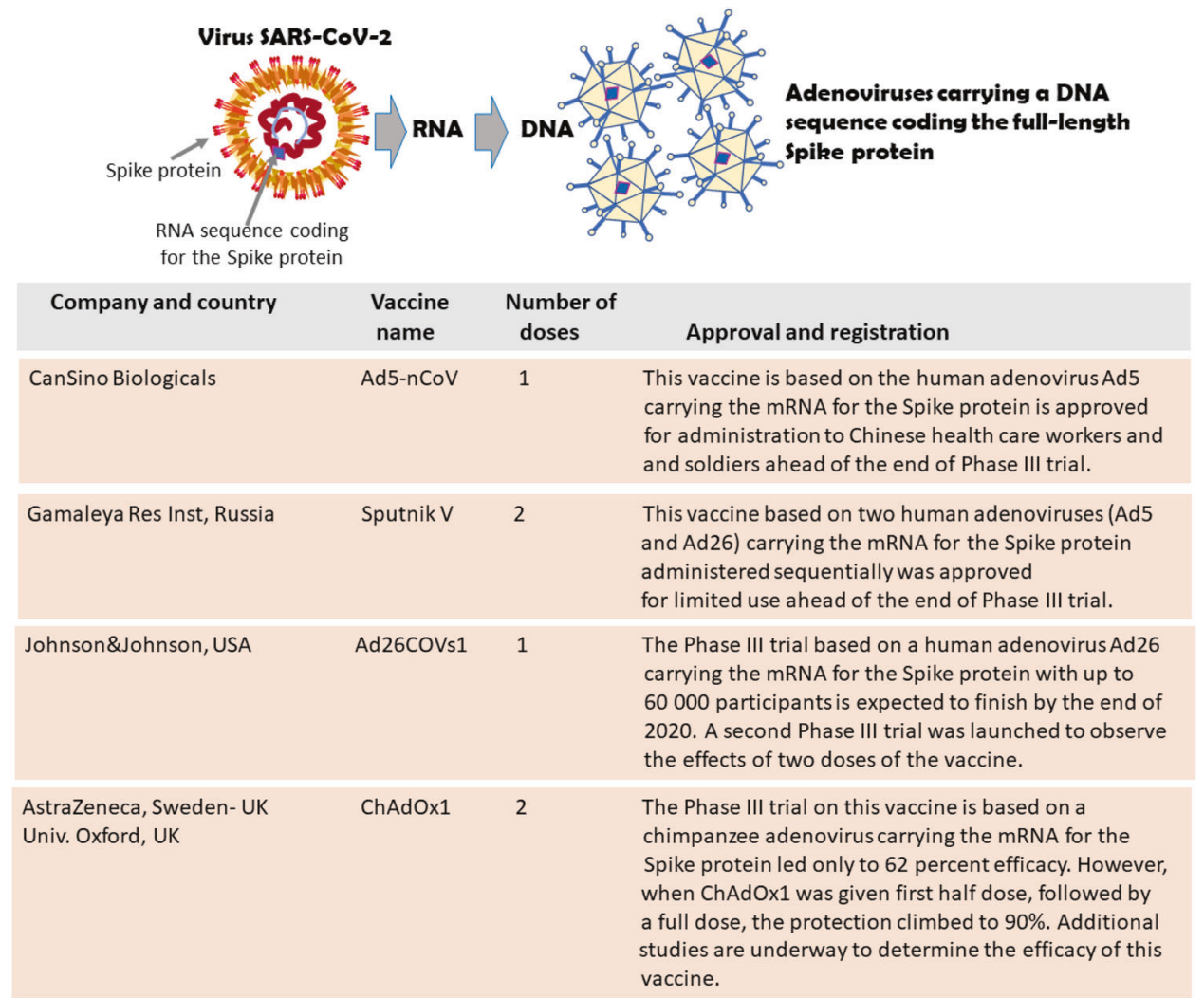

ReiThera, Italy.

some extent, they may induce a more robust immune response [14].

Also in these vaccine projects, the target antigen coded by the DNA is mostly, if not only, the Spike protein, its variants, or its fragments. Commonly, these virus-based vaccines are injected intramuscularly. However, there are numerous and interesting projects aiming at administering the vaccine into the nose by inhalation. If effective, the candidate vaccine could induce a mucosal immunity capable of neutralizing the virus, thus inhibiting its ability to enter the human body.

\section{Frontrunners}

There are very numerous vaccine projects based on viral vectors that are already in advanced clinical trials. Four of those are currently in Phase III trial or approved for limited use (Fig. 5). The vaccine DNA is inserted inside:

a. Engineered non-replicating virus vectors

1. Chimpanzee adenovirus: AstraZeneca, Univ. Oxford, Sweden-UK-Italy (Fig. 5), that is also testing a vaccine inhaled form not yet in Phase III trial;

2. Gorilla adenovirus:v
3. Human adenoviruses:

- CanSino, China (Fig. 5);

- Johnson\&Jonhson, US, (Fig. 5);

- Acad Mil Med Sci, China

- Gamaleya Res Inst, Russia: this vaccine based on two human adenoviruses injected one after the other has been approved for limited use (Fig. 5).

4. Adenoviruses specifically modified for nasal spray:

- Beijing Wantai Biol Pharm Enterprise, China;

- Acad Mil Sci, China, two projects;

- Bharat Biotech-Washington Univ, India-US;

- AstraZeneca, Sweden-UK;

- Altimmune, US.

5. Other viruses

b. Engineered replicating virus vectors

1. Injected intramuscularly:

- Measles virus, Merck, US;

- Vesicular Stomatitis Virus.

2. Influenza virus administered by nasal spray:

- Influenza virus: 
Univ Hong Kong;

Valavax-Abogn, China;

Beijin Vantal Biol Pharm, China.

\section{A few other technological platforms}

1. Symvivo, Canada: A Phase I human trial is underway with orally administered Bifidobacterium probiotic, engineered to carry the DNA encoding the Spike protein.

2. Immunomonitor, Canada: A Phase I/II human trial is underway with heat-inactivated plasma from donors with COVID-19.

3. Aivita Biomedical, US: A Phase I/II human trial is underway with the patient's dendritic cells modified to express SARS-CoV-2 antigens.

4. Shenzhen Geno-Immune Medical, China: A Phase I human trial is underway with dendritic cells engineered to express SARS-CoV-2 proteins.

\section{Selected vaccines in clinical evaluation}

As this report was being drafted, about eleven candidate vaccines have entered the most advanced phase of clinical assessment. For five of these, results obtained in Phase II have been made available in peer-reviewed journals [15-21]. Here we will briefly comment on available information based on these publications. The number of subjects enrolled ranged from 100 to 1077 and the study design was usually a single arm. The Oxford/AstraZeneca Phase II trial included 1077 patients randomized to an irrelevant meningococcus vaccine or the adenovirus-based SARS-CoV2 vaccine [16]. Selected common findings include: activation of innate immunity, as revealed by local and systemic inflammation; induction of antibodies, including neutralizing antibodies; induction of type 1T cell responses with Interferon-gamma production. Type 1 immune responses are considered a cornerstone of antiviral immunity. At least in the most extensive study, the AstraZeneca vaccine trial, induction of type 2 potentially deleterious $\mathrm{T}$ cell responses were not observed. Collectively, these results are encouraging but suffer from limitations, extensively discussed in some of the reports [16]. Follow-up was limited ( $<60$ days) and the duration of persistence of immunological memory remains to be determined. The actual significance of immunological parameters for protection remains to be defined. Aging is associated with loss of immunological memory, reduced repertoire of responses, and increased inflammatory tone [22]. As a consequence, for instance, vaccines designed for the elderly have been introduced for influenza. Therefore, it appears of particular interest that in a randomized Phase $2 / 3$ trial enrolling 560 participants of which 240 were aged 70 years or older, the Oxford/AstraZeneca vaccine was able to elicit an effective production of SARS-CoV-2 neutralizing antibodies and to elicit $\mathrm{T}$ cellmediated reactivity in all vaccinated participants, even in adults over 70 years of age. Indeed, the volunteers of this age group showed a reduced reactogenicity [17]. Moreover, to assist with the interpretation of the data, the full details of this trial operation procedure have been made available [18]. A Phase 1 randomized trial enrolling 195 participants to compare the immunogenicity and reactivity of Pfizer BioNTech liposome-mRNA vaccines (BNT162b1 and BNT162b2) both vaccine candidates elicited similar dosedependent SARS-CoV-2 antibody titers in young adults and those between 65 and 85 years of age [23]. In a Phase I dose-escalation trial, the Moderna mRNA-1273 liposome mRNA vaccine administered to 40 older adults who were stratified according to age (56-70 and over 70) elicited titers of neutralizing antibody similar to those previously reported among vaccine recipients between the ages of 18 and 55 years [24].

An interim analysis of ongoing large Phase $2 / 3$ trials in UK and Brazil confirmed that the Oxford/AstraZeneca vaccine has an acceptable safety profile. In the participants enrolled in the UK and Brazil who received the vaccine standard dose, consistently vaccine efficacy was $62 \%$. By contrast, in a subset of participants in the UK trial who received a half vaccine dose as their first dose and a standard dose as their booster dose, vaccine efficacy was $90 \%$ [25]. Despite the enthusiasm for this finding, which puts the Oxford/AstraZeneca vaccine at the efficacy level of those of Pfizer-BioNTech and Moderna (Fig. 4) the interpretation of this results ore not yet established. It is not uncommon that different doses between the first and second vaccination may potentiate the intensity of the induced immune response. Alternatively, a lower first dose of the vaccine may not elicit a strong reaction against the chimpanzee adenovirus (Fig. 5). In the absence of a strong antibody response, the adenovirus could more effectively deliver the mRNA during the booster vaccination. This is also the rationale for the Sputnik V vaccine, which uses a different type of adenovirus in the first vaccination and the booster injection [9]. These puzzling data are spurring the UK vaccine task force to plan a "mix and match" trial that will combine both the Oxford/AstraZeneca and the PfizerBionTHech vaccine to evaluate if the two vaccines together produce a stronger immune response The two vaccines may trigger slightly different immune responses that could mutually enhance each other [26].

Available clinical documentation on the Sputnik V vaccine prepared by the Gamaleya Research Institute, registered in Russia is based on a small clinical study involving 76 volunteers [9, 27, 28]. However, a large post- 
registration clinical trial involving more than 40,000 people in Russia and is currently ongoing. In November 2020, joining Pfizer-BioNTech, Moderna, and AstraZeneca press releases, Gamaleya Research Institute announced that an interim analysis based on 18,794 volunteers who received both the first and second dose of the Sputnik V vaccine showed a $91.4 \%$ efficacy seven days after the second dose. Two weeks later protective efficacy was over 95\% [29].

Vaccine reactogenicity refers to a subset of inflammatory reactions that occur soon after vaccination. While no safety concerns appear to be related to the administration of Pfizer - BioNTech, Moderna, Oxford/AstraZeneca, and Gamalaya Res. Inst vaccine administration, the frequency of inflammatory reactions is higher than that commonly observed with flu vaccines [30]. Of the two candidate vaccines initially tested by Pfizer - BioNThec, the BNT162b1 coding for the Receptor-Binding Domain (RBD) of the Spike protein (see Fig. 5B) was dismissed since it was more reactogenic than the BNT162b2 coding for the entire Spike protein $[23,30,31]$. In the AstraZeneca trials, the ChAdOx1 candidate vaccine induced a similar immune response across all age groups, while it was less reactogenic in older adults than in younger adults [16]. Early markers for reactogenicity, including the humoral innate immunity molecule PTX3, may help early assessment of activation of innate immunity and reactogenicity [32-34]. COVID-19 has been associated in a fraction of patients with the development of autoimmune reactions blocking the Interferon pathway or eliciting thrombosis [32-34]. Although there have been no major problems in patients suffering from autoimmune disorders, further studies are needed to assess the impact of COVID-19 and vaccines on autoimmunity including myasthenia gravis $[35,36]$.

\section{Efficacy assessment}

Even if incomplete, the list of ongoing clinical trials shown above gives an idea of the grandiose scientific, technical, and organizational effort that is currently underway. The administration of the new vaccine makes it possible to understand whether the vaccine induces a significant immune response and whether its administration causes clear adverse events. The candidate vaccines which are considered worthy of study in complex Phase III human trials are the ones that, on a limited number of volunteers in Phase I and II clinical trials, caused minor short-term side effects while inducing a good production of antibodies capable of neutralizing the infectivity of SARS-CoV-2 (neutralizing antibodies), and, in some cases, a significant $\mathrm{T}$ cell activation. Even if the assays to evaluate the immune response vary vastly, protein-based vaccines appear to elicit the strongest antibody response [14].

The real evaluation of the effectiveness of the new vaccine is based on Phase III randomized controlled trials that compare the incidence of COVID-19 in large groups of vaccinated and non-vaccinated people. This evaluation will determine whether one, several or none of the new COVID19 vaccines protects effectively or only marginally and if its administration is associated with important collateral events. The situation may arise where the scientific efforts and the huge funding investments will result in the development of fifteen or more frontrunners that will be registered for general use and compete head-to-head.

\section{Comparative evaluations}

-Mirror, mirror on the wall, who's the fairest of them all? Data from Phase III studies will provide an excellent indication of the efficacy, limits, and safety of the candidate vaccines. However, only a few of the long list of vaccines will be directly compared. The WHO has put forward Solidarity efficacy trials open to vaccines from every country and has made public detailed criteria on how to prioritize vaccine efficacy [37]. The vaccines included in Operation Warp Speed, the US government's private-public partnership to support COVID-19 vaccines, have harmonized efficacy protocols to streamline oversight and run immunological analyses in central labs for achieving a direct data comparison [38]. But what about all the other projects? Although any vaccine that will be registered in Europe and the USA will be assessed on a long series of data for its efficacy and safety, it will likely be a long time before industrial policies and national political issues could allow a solid comparative assessment of the efficacy of the various vaccines. Quantitative comparisons of the efficacy of different vaccines in inducing immune responses are hampered by the lack of international standards. For instance, there is no international standard at the moment for titrating IgG anti-Spike serum antibodies, let alone for neutralization assays or $\mathrm{T}$ cell responses. This current technological limitation makes it difficult to compare immune responses elicited by vaccines in different trials.

It is also conceivable that "the best" COVID-19 vaccine could not exist at all, as vaccines developed on distinct technological platforms could induce different forms of immunity, each of them appropriate in different environmental and human contexts. The polio vaccine provides an interesting example. The injectable killed Salk vaccine is turning out to be appropriate for the industrialized world: it is safe and effective in areas of the world where polio no longer exists. By contrast, the attenuated Sabin vaccine, more effective and easier to be 
administered orally, is appropriate for the developing world where the wild virus is still circulating. Thus, the evolution of the pandemic could make some vaccines more appropriate in different geographic contexts or for different clusters (e.g., infants, elderly) of the human population [39]. It is quite possible that the sequential arrival of subsequent wages of vaccines could increase and make more appropriate the protection initially induced by the first vaccines.

\section{The unknowns}

It is expected that the first vaccines that will be made available will significantly contribute to the normalization of social life, even if their arrival will be accompanied by a long list of unknowns. Most of these unknowns are inherently associated with the dramatic pressure brought on by the pandemic and the reactive speed at which these vaccines are being developed. The passage of time along with the luxury of being able to develop more detailed studies will lead to the clarification of many questions left open by the first Phase III studies.

Only time will tell how long the vaccine-elicited protection will last and how frequent the booster injections should be administered to keep the protection fully active. Over time, population data will better clarify what kind of protection the various vaccines can induce. Will the vaccine protect people only from the mildest form of COVID-19, or will it be one that prevents serious complications and reduces mortality? Often Phase III trials are designed to test whether the vaccines reduce cases of symptomatic COVID-19, not cases of severe diseases, such as those that require hospitalization and can end in death [40].

Who will be protected is another crucial question that only more detailed studies can answer. Phase III trials currently underway are mainly focused on a healthy population. A vaccine judged to be effective on this population may not work equally well in elderly individuals, frail, and other at-risk persons. Even if children are not a high-risk group, as the schools reopen, the transmission of the virus could take place among students, their parents, and school staff [41]. Vaccinating children could help reopen society, ensuring that schools do not become hot spots. Current trials, such as the AstraZeneca phase III in the USA now include children. Pregnant women are another high-risk group since they have a higher risk of being admitted to an intensive care unit and of requiring mechanical ventilation [42]. Will the vaccines also be able to prevent the spread of the disease? In effect, while protecting from the clinical disease vaccines might not reduce virus transmission [43]. Among many other unknowns that can be solved over time, there is the question of whether the arrival of vaccines will be able to create the herd immunity capable of controlling the spread of SARS-CoV-2 [44]. Complex social policy issues and the acceptance or rejection of vaccination by the population will significantly affect the possibility of achieving this crucial goal.

\section{Risks associated with fast track vaccine evaluation}

The administration of a new vaccine must always be carefully associated with a rigorous study of its safety. This is particularly important because a vaccine is not a drug for sick people at risk of dying, but rather a treatment that is given to those who are well to prevent the risk of falling ill [1].

The race to develop a COVID-19 vaccine is not only justified but necessary. However, the time required to evaluate the dangers and risks that may arise from a new vaccine must be included in its development. In some cases, vaccines prepared against other coronaviruses or other viruses have worsened the disease [45] and have induced $\mathrm{T}$ helper 2-type immunopathology [46]. These issues must be carefully evaluated and excluded before a new COVID-19 vaccine is distributed to combat the pandemic or its subsequent outbreaks. These basic considerations take on special importance when inappropriate political pressures may lead to accelerating the evaluation of vaccine safety. Claiming to have won the race to develop a COVID-19 vaccine or the distribution of a candidate vaccine to clusters of the population before all data from clinical trials are obtained and carefully analyzed can be dangerous and erode trust in both the vaccine and regulatory bodies. In this weird contest, the pledges put forward both by pharmaceutical companies and the director of the US Objective Warp Speed [13] to keep rigorous efficacy and safety standards as an absolutely central issue in COVID-19 vaccine development are reassuring.

\section{SARS-CoV-2 genetic instability and implications for vaccine development}

At the time of writing, there are over 5000 complete sequencing data of viral isolates reported, and at least three peer-reviewed papers available [47-49]. SARS-CoV-2 is relatively stable and no evidence has been obtained that an attenuated virus has spread globally. For instance, sequencing of 346 virus isolates in the Lombardy region has shown that the spike protein does not undergo mutations including at glycosylation sites. The relative stability of SARS-CoV-2, unlike HIV, provides a strong rationale for 
vaccine development. However, following the introduction of an effective vaccine(s), the mutant virus may appear and have a selective advantage, thus posing the issue of adapting the vaccine strategy.

\section{Pathogen-agnostic protection conferred by COVID-19 unrelated vaccines and anti-SARS-CoV-2 vaccines}

There is strong evidence that selected vaccines confer what has been referred to as pathogen-agnostic protection against infectious agents unrelated to the one specifically targeted [50-53].

For instance, the measles and BCG vaccines are strongly associated with a reduced incidence of unrelated respiratory tract infections [54]. The mechanisms of pathogen-agnostic protection are complex and include avoidance of virusinduced immunosuppression and increased effectiveness ("training") of innate immunity. The evidence for pathogenagnostic protection and training of innate immunity is strong for BCG [52]. Moreover, it has been speculated that the intense vaccination calendar for children contributes to their as yet unexplained resistance to COVID-19. Based on these considerations several prospective clinical trials are ongoing aimed to assess the value of BCG for instance for the protection of health care workers. At this stage, usage of BCG as a preventive measure against COVID-19 cannot be recommended outside of clinical trials [52]. However, available information suggests that vaccines such as influenza, pneumococcus, and herpes in the elderly represent a general training strategy for innate and adaptive immunity. Moreover, it will be important to assess whether antiSARS-CoV-2 vaccines increase immunological fitness as this may be relevant to fight escape variants.

\section{Production and ethical issues}

Once the new vaccine has been validated, subsequent problems will be related to its production and distribution. Technological, organizational, regulatory, and economic problems will have to be overcome. The industrial technology needed to scale up the production to a billion doses will depend on which kind of vaccine will work best. Initially, it might not be physically possible to make enough vaccines for the world's population, although, various vaccines are already in production without being sure that they will be registered and distributed. Besides, political and economic constraints may limit vaccine access to the country that produces it or to the countries that can afford to pay for it. To make the new vaccines available to the global population will be challenging [55]. The problem of a fair distribution of the vaccine in all the nations of the world is much discussed and various initiatives are about to be implemented by several nations and international organizations [14, 56-59]. To ensure equitable access to future COVID-19 vaccines the Coalition for Epidemic Preparedness Innovations (CEPI), The Global Alliance for Vaccines and Immunization (GAVI), and WHO have launched the COVID-19 Vaccines Global Access (COVAX) Facility, a global risk-sharing mechanism for pooled procurement and equitable distribution of eventual COVID-19 vaccines [60].

\section{Biohackers}

The enthusiasm to design different COVID-19 vaccines is also plaguing the biohacking community. Biohacking is a "do-it-yourself biology", a somewhat romantic biotechnological social movement in which individuals and small organizations are involved in transforming both life sciences and information systems using low cost, open-source alternatives, and open-access tools, claiming independence from both academic and corporate institutions. In recent weeks, several biohackers have taken part in online biology forums to help investigate potential vaccines and innovative methods of testing them, often on the vaccine inventors themselves. The hunt for a free, open science Coronavirus vaccine is on [61].

\section{Vaccine mistrust}

Along with the progressive development of modern vaccines, opinion movements against vaccination have flourished in the Western world. Currently, the news on antiCOVID-19 vaccines have further ignited opposition protests $[62,63]$. In addition to the movements openly opposed to vaccination, several polls show that a significant percentage of people in the Western countries would be hesitant or contrary to take a COVID-19 vaccine once it is approved. The chief concern among those surveyed was that the vaccine approval process would move too quickly without taking the time to properly establish safety and effectiveness. Certainly, the intrusion of political issues into the pace of the vaccine evaluation process does not help to build confidence in new vaccines. However, by the time vaccines are registered and made available, data on their efficacy and safety will have been carefully reviewed by several national and international agencies. On the other hand, these vaccines will have had a short documentation history and might elicit hypothetical side effects after a long time, that could not have been previously appreciated.

This is, however, a conjectural situation. Instead, it is likely that, once the efficacy of a COVID-19 vaccine will be demonstrated, much of the hesitation about this vaccination will quickly vanish. It will be commonly apparent that a marked reduction in the risk of dying from 
COVID-19 largely compensates for the risk of hypothetical late side effects.

\section{Fraudulent vaccines}

Attempts to obtain these vaccines at any cost are almost mirroring the vaccine mistrust. The illegal purchase of Chinese vaccines, and probably Chinese fake vaccines, is starting to be advertised on some internet sites [64]. Certainly, the possibility of the inhabitants of the eastern Chinese city of Jiaxing involved in essential works to buy the CoronaVax vaccine made by Sinovac Biotech which is still being in Phase III trial does not favor the control of this dangerous trade (Fig. 1).

\section{Diverse vaccine platforms}

The pandemic drama has prompted many scientists around the world to design possible alternative COVID-19 vaccines. Thus, in addition to the large number of official projects enlisted by WHO [4], numerous university laboratories and small biotech firms are studying fresh vaccines. This clever intellectual and technological effort provides myriads of diverse projects, some of which could become important if front runner projects will confer only partial protection or work poorly in certain clusters of the population. High costs and other barriers might make some of the front runner vaccines unsuitable for wide-scale deployment in lower-income countries [11, 12, 39]. For example, bacteriophage-based vaccines that infect nose and throat microbes and make them produce the Spike protein, or other vaccines that could be administered by nasal insufflation or by mouth appear to be stimulating alternatives. It would really be a great achievement to develop a vaccine that can induce an effective immunity on the mucosal surfaces: it could impede the viral infection and the virus spread through respiratory droplets.

The success and approval of the first COVID-19 vaccines should not detract from the enthusiasm and practical possibility of planning new studies leading to the development and production of second and third-generation vaccines $[11,12]$ as well as the design of different types of clinical trials [65]. Indeed, COVID-19 eradication is going to be a long and winding road that will not finish once we have the first vaccine available.

\section{Conclusions}

As we are completing this briefing, the COVID-19 vaccines are on the way. Currently, fresh data from Phase III trials are permitting vaccine registration. Soon after, few vaccines based on different technologies will be mandatory or made available for selected clusters of the population of China, Russia, the United States, and Europe.

Thus, the landscape of the pandemic is taking on completely different features. We will know if vaccines to efficaciously control the SARS-CoV-2 virus spreading remain a far goal or if they are already here. In the latter case, the next burning issue will be vaccine availability and its equitable distribution in all areas of the world. Predatory national politics aimed at ensuring that the first vaccine doses are made available to the population of their nation will clash with attempts of many international organizations to set up a more fair distribution in all countries of the world. This noble effort is severely contrasted by the political significance that the COVID-19 vaccine is assuming. The political leader or the country that produces a first salvific vaccine can exploit it to affirm its ability to protect its citizens as well as the inhabitants of friendly countries. The vaccine, thus, may become an inappropriate measure of power $[48,59]$. How many seeds of disease, despair, and death will difficult access to the vaccine sow among the people of the earth? Cynically, it could just be the relentless predatory rush to grab the first doses of the vaccine that could produce a fair distribution of surplus vaccines to the less rich nations in a relatively short time [65].

Finally, it is our tenet that in the long run more than one vaccine will be needed to ensure equitable global access, protection of diverse subjects, and immunity against viral variants.

Acknowledgements We thank Prof. Giuseppina Barsacchi for critically reading the manuscript. Alberto Mantovani acknowledges support by the Dolce \& Gabbana fashion company.

The COVID-19 Commission of Accademia Nazionale dei Lincei, Rome Guido Forni ${ }^{4}$, Alberto Mantovani ${ }^{4}$, Lorenzo Moretta ${ }^{4}$, Rino Rappuoli ${ }^{4}$, Giovanni Rezza ${ }^{4}$, Arnaldo Bagnasco ${ }^{4}$, Giuseppina Barsacchi $^{4}$, Giovanni Bussolati ${ }^{4}$, Massimo Cacciari ${ }^{4}$, Pietro Cappuccinelli ${ }^{4}$, Enzo Cheli ${ }^{4}$, Renato Guarini ${ }^{4}$, Massimo Livi Bacci ${ }^{4}$, Marco Mancini $^{4}$, Cristina Marcuzzo ${ }^{4}$, Maria Concetta Morrone ${ }^{4}$, Giorgio Parisi $^{4}$, Gianfranco Pasquino ${ }^{4}$, Carlo Patrono ${ }^{4}$, Alberto Quadrio Cur$\mathrm{zio}^{4}$, Giuseppe Remuzzi ${ }^{4}$, Alessando Roncaglia ${ }^{4}$, Stefano Schiaffino, Paolo Vineis ${ }^{4}$

${ }^{4}$ The Commissione COVID-19, Accademia Nazionale dei Lincei, Rome, Italy

\section{Compliance with ethical standards}

Conflict of interest GF declares no conflict of interest. AM has obtained lecture and consulting fees from Astra Zeneca, Pfizer and Janssen.

Publisher's note Springer Nature remains neutral with regard to jurisdictional claims in published maps and institutional affiliations. 


\section{References}

1. Forni G, Mantovani A, Moretta L, Rezza G Vaccines. Accademia Nazionale dei Lincei. 2018. https://www.lincei.it/it/article/i-va ccini-vaccines-position-paper.

2. Piccoli L, Park YJ, Tortorici MA, Czudnochowski N, Alexandra $\mathrm{C}$, Walls AC, et al. Mapping neutralizing and immunodominant sites on the SARS-CoV-2 spike receptor-binding domain by structure-guided high-resolution serology, Cell. 2020;183: $1024-42$.

3. Lurie N, Saville M, Hatchett R, Halton J. Developing Covid-19 Vaccines at pandemic speed. $N$ Engl J Med. 2020;382: 1969-73.

4. WHO. Draft landscape of COVID-19 candidate vaccines. 2020. https://www.who.int/publications/m/item/draft-landscape-of-

COVID-19-candidate-vaccines.

5. Akst J. COVID-19 vaccine frontrunners. The Scientist. 2020. https://www.the-scientist.com/news-opinion/covid-19-vaccinefrontrunners-67382.

6. Pfizer, Pfizer and BioNTech conclude Phase 3 study of COVID19 vaccine candidate, meeting all primary efficacy endpoints. 2020. https://www.pfizer.com/news/press-release/press-releasedetail/pfizer-and-biontech-conclude-phase-3-study-covid-19-va ccine.

7. Moderna, Moderna's COVID-19 vaccine candidate meets its primary efficacy endpoint in the first interim analysis of the phase 3 COVE study. 2020. https://investors.modernatx.com/node/ $10316 /$ pdf.

8. AstraZeneca, AZD1222 vaccine met primary efficacy endpoints in preventing COVID-19. 2020. https://www.astrazeneca.com/ media-centre/press-releases/2020/azd1222hlr.html.

9. Sputnik V. The first registered vaccine against COVID-19. https:// sputnikvaccine.com/

10. Cohen J. Vaccine wagers on coronavirus surface protein pay off. Science. 2020;370:894-5.

11. Callaway E. The underdog coronavirus vaccines that the world will need if front runners stumble. Nature. 2020;585:332-3.

12. Cohen J. First vaccine may stymie hunt for better ones. Science. 2020;370:389-90.

13. Cohen J. Leader of U.S. vaccine push says, he'll quit if politics trumps science. Science. 2020. https://doi.org/10.1126/science. abe6380.

14. Krammer F. SARS-CoV-2 vaccines in development. Nature. 2020;586:516-27.

15. Xia S, Duan K, Zhang Y, Zhao D, Zhang H, Xie Z, et al. Effect of an inactivated vaccine against SARS-CoV-2 on safety and immunogenicity outcomes: interim analysis of 2 randomized clinical trials. JAMA. 2020;324:951-60.

16. Folegatti PM, Ewer KJ, Aley PK, Angus B, Becker S, BelijRammerstorfer S, et al. Safety and immunogenicity of the ChAdOx1 nCoV-19 vaccine against SARS-CoV-2: a preliminary report of a phase 1/2, single-blind, randomised controlled trial. Lancet. 2020;396:467-78.

17. Ramasamy MN, Minassian AM, Ewer KJ, Flaxman AL, Folegatti PM, Owens DR, et al. Safety and immunogenicity of ChAdOx1 $\mathrm{nCoV}-19$ vaccine administered in a prime-boost regimen in young and old adults(COV002): a single-blind, randomised, controlled, phase 2/3 trial. Lancet. 2020;396. https://doi.org/10.1016/S01406736(20)32466-1.

18. Pollard AJ. Notice of addendum to article reporting Oxford trial of ChAdOx1nCoV-19 vaccine. Lancet. 2020;396:e89.

19. Poland GA, Ovsyannikova IG, Kennedy RB. SARS-CoV-2 immunity: review and applications to phase 3 vaccine candidates. Lancet. 2020;396:1595-606.
20. Jackson LA, Anderson EJ, Rouphael NG, Roberts PC, Makhene $\mathrm{M}$, Coler RN, et al. An mRNA vaccine against SARS-CoV-2 preliminary report. N Engl J Med. 2020;383:1920-31.

21. Zhu FC, Guan XH, Li YH, Huang JY, Jiang T, Hou LH, et al. Immunogenicity and safety of a recombinant adenovirus type-5vectored COVID-19 vaccine in healthy adults aged 18 years or older: a randomised, double-blind, placebo-controlled, phase 2 trial. Lancet. 2020;396:479-88.

22. Bottazzi B, Riboli E, Mantovani A. Aging, inflammation and cancer. Semin Immunol. 2018;40:74-82.

23. Walsh EE, Frenck RW Jr, Falsey AR, Kitchin N, Absalon J, Gurtman A, et al. Safety and immunogenicity of two RNA-based covid-19 vaccine candidates. New Engl J Med. https://doi.org/10. 1056/NEJMoa2027906.

24. Anderson EJ, Rouphael, NG, Widge AT, Jackson LA, Roberts PC, Makhene M, et al. Safety and immunogenicity of SARSCoV-2 mRNA-1273 vaccine in older adults. New Engl J Med. 2020. https://doi.org/10.1056/NEJMoa2028436.

25. Voysey M, Ann S, Clemens C, Madhi SA, Weckx LY, Folegatti $\mathrm{P}$, et al. Safety and efficacy of the ChAdOx $1 \mathrm{nCoV}-19$ vaccine (AZD1222) against SARS-CoV-2: an interim analysis of four randomised controlled trials in Brazil, South Africa, and the UK. Lancet. 2020. https://doi.org/10.1016/S0140-6736(20)32661-1.

26. Boseley S. UK trial to mix and match Covid vaccines to try to improve potency. The Guardian. 2020. https://www.theguardian. com/world/2020/dec/08/covid-mixed-vaccine-trial-likely-tobegin-in-uk-next-month.

27. Cohen J. Russia's approval of a COVID-19 vaccine is less than meets the press release, Science. 2020. https://doi.org/10.1126/ science.abe2848.

28. Callaway E. Russia's fast-track coronavirus vaccine draws outrage over safety. Nature. 2020;584:334-335.

29. Sputnik V. Second interim analysis of clinical trial data. 2020. https://sputnikvaccine.com/newsroom/pressreleases/secondinterim-analysis-of-clinical-trial-data-showed-a-91-4-efficacy-forthe-sputnik-v-vaccine-on-d/.

30. Wadman M. Fever, aches from Pfizer, Moderna jabs aren't dangersous but may be intense for some. Science. 2020. https://www. sciencemag.org/news/2020/11/fever-aches-pfizer-moderna-jabs-a ren-t-dangerous-may-be-intense-some.

31. Pfizer. Pfizer and Biontech choose lead mRNA vaccine candidate against COVID-19 and commence pivotal Phase $2 / 3$ global study. 2020. https://www.pfizer.com/news/press-release/press-releasedetail/pfizer-and-biontech-choose-lead-mrna-vaccine-candidate-0

32. Brunetta E, Folci M, Bottazzi B, De Santis M, Gritti G, Protti A, et al. Macrophage expression and prognostic significance of the long pentraxin PTX3 in COVID-19. Nature Immunol. 2020. https://doi.org/10.1038/s41590-020-00832-x.

33. Bottazzi B, Santini L, Savino S, Giuliani MM, Dueñas Diez A, Mancuso $G$, et al. Recognition of Neisseria meningitidis by the long pentraxin PTX3 and its role as an endogenous adjuvant. PLoS ONE. 2015;10:e0120807.

34. Weiner J, Lewis DJM, Maertzdorf J, Mollenkopf H-J, et al. Characterization of potential biomarkers of reactogenicity of licensed antiviral vaccines: randomized controlled clinical trials conducted by the BIOVACSAFE consortium. Sci Rep. 2019;9:20362.

35. Novelli L, Motta F, De Santis M, Ansari AA, Gershwin ME, Selmi C. The JANUS of chronic inflammatory and autoimmune diseases onset durin COVID-19 - a systematic review of the literature. J Autoimmunity. 2020;117:102592. https://doi.org/10. 1016/j.jaut.2020.102592.

36. Muppidi S, Guptill JT, Jacob S, Li Y, Farrugia ME, Guidon AC, et al. COVID-19-associated risks and effects in myasthenia gravis (CARE-MG). Lancet Neurol. 2020;19:970-1. 
37. WHO. Access to Covid-19 Tools (ACT) Accelerator -A Global Collaboration to accelerate the development, production and equitable access to new COVID-19 diagnostics, therapeutics and vaccines. 2020. https://www.who.int/who-documents-detail/a ccess-to-covid-19-tools-(act)-accelerator.

38. HHS.gov. Trump administration announces framework and leadership for 'Operation Warp Speed'. 2020. https://www.hhs.gov/a bout/news/2020/05/15/trump-administration-announces-framew ork-and-leadership-for-operation-warp-speed.

39. Tagliabue A, Forni G. COVID-19: who will produce the vaccine? Accademia Nazionale dei Lincei. 2020. https://www.lincei.it/it/a rticle/covid-19-who-will-produce-vaccine.

40. Mallapaty S, Ledford H. COVID-vaccine results are on the way and scientists' concerns are growing. Nature. 2020;586:16-17.

41. Boehmer TK, DeVies J, Caruso E, van Santen KL, Tang S, Black CL, et al. Changing age distribution of the COVID-19 Pandemic United States, May-August 2020. MMWR Morb Mortal Wkly Rep. 2020;69:1404-9.

42. Delahoy MJ, Whitaker M, O'Halloran A, Chai SJ, Kirley PD, Alden N, et al. Characteristics and maternal and birth outcomes of hospitalized pregnant women with laboratory-confirmed COVID19 - COVID-NET, 13 States, March 1-August 22, 2020. MMWR Morb Mortal Wkly Rep. 2020;69:1347-54.

43. Peiris M, Leung GM. What can we expect from first-generation COVID-19 vaccines? Lancet. 2020;396:1467-9.

44. Saad-Roy CM, Wagner CE, Baker RE, Morris SE, Farrar J, Graham AL, et al. Immune life history, vaccination, and the dynamics of SARS-CoV-2 over the next 5 years. Science. 2020;370:811-8.

45. Jiang S. Don't rush to deploy COVID-19 vaccines and drugs without sufficient safety guarantees. Nature. 2020;579:321.

46. Tseng CT, Sbrana E, Iwata-Yoshikawa N, Newman PC, Garron T, Atmar RL, et al. Immunization with SARS coronavirus vaccines leads to pulmonary immunopathology on challenge with the SARS virus. PloS ONE. 2012;7:e35421.

47. Young BE, Fong SW, Chan YH, Mak TM, Ang LW, Anderson DE, et al. Effects of a major deletion in the SARS-CoV-2 genome on the severity of infection and the inflammatory response: an observational cohort study. Lancet. 2020;396:603-11.

48. Grubaugh ND, Hanage WP, Rasmussen AL. Making sense of mutation: what D614G means for the COVID-19 pandemic remains unclear. Cell. 2020;182:794-5.

49. Li Q, Wu J, Nie J, Zhang L, Hao H, Liu S, et al. The impact of mutations in SARS-CoV-2 spike on viral infectivity and antigenicity. Cell. 2020;182:1284-94.e1289.

50. Sala G, Miyakawa T. Association of BCG vaccination policy with prevalence and mortality of COVID-19. medRxiv. 2020. https:// doi.org/10.1101/2020.03.30.20048165.
51. Shet A, Ray D, Malavige N, Santosham M, Bar-Zeev N Differetial COVID-19-attributable mortality and BCG vaccine use in countries. medRxiv. 2020. https://doi.org/10.1101/2020.04.01. 20049478doi.

52. Mantovani A, Netea M. Trained Innate Immunity: epigenetics and Covid-19. N. Engl J Med. 2020;383:1078-80.

53. Giamarellos-Bourboulis EJ, Tsilika M, Moorlag S, Antonakos N, Kotsaki A, Dominguez-Andres J, et al. Activate: randomized clinical trial of BCG vaccination against infection in the elderly. Cell. 2020;183:315-23.e319.

54. de Vrieze J Can a century-old TB vaccine steel the immune system against the new coronavirus? Science. 2020. https://doi. org/10.1126/science.abb8297.

55. Khamsi R. If a coronavirus vaccine arrives, can the world make enough? Nature. 2020;580:578-80.

56. Kupferschmidt K. Vaccine nationalism' threatens global plan to distribute COVID-19 shots fairly. Science. 2020. https://doi.org/ 10.1126/science.abe 0601 .

57. Gates B. When a COVID-19 vaccine is ready, this group will make sure the whole world can access it. Gates Foundation. 2020. https://www.gatesfoundation.org/TheOptimist/Articles/corona virus-gavi.

58. Accad.Naz.Lincei. COVID-19: fair access to vaccines. 2020. https://www.lincei.it/it/article/covid-19-fair-access-vaccines.

59. Accad. Naz. Lincei. COVID-19: Equiteable distribution of vaccines. 2020. https://www.lincei.it/it/article/equitable-distributionvaccines.

60. GAVI. 2020. https://www.g5avi.org/covax-facility.

61. Heidt A. Self experimentation in the time of COVID-19. The Scientist. 2020. https://www.the-scientist.com/news-opinion/selfexperimentation-in-the-time-of-covid-19-67805.

62. Cornwall W. Officials gird for a war on vaccine misinformation. Science. 2020;369:14-5.

63. Wadman M. Vaccine opponents are gaining in Facebook 'battle for hearts and minds,' ne w map shows. Science. 2020. https://doi. org/10.1126/science.abc7822.

64. U.S. Food \& Drug Administration. Beware of fraudolent coronavirus tests, vaccines and treatments. 2020. https://www.fda. gov/consumers/consumer-updates/beware-fraudolent-coronavirustests-vaccines-and-treatments.

65. Bollyky TJ, Gostin LO, Hamburg MA. The equitable distribution of COVID-19 therapeutics and vaccines. JAMA. 2020;323:2462-3.

66. Hoffmann M, Weber HK, Schroeder S, Krüger N, Herrler T, Erichsen S, et al. SARS-CoV-2 cell entry depends on ACE2 and TMPRSS2 and is blocked by a clinically proven protease inhibitor. Cell. 2020;181:271-80. 